\title{
Plasma simulation studies using multilevel physics models*
}

\author{
W. Park, , a) E. V. Belova, G. Y. Fu, and X. Z. Tang \\ Princeton University Plasma Physics Laboratory, Princeton, New Jersey \\ H. R. Strauss \\ New York University, New York, New York \\ L. E. Sugiyama \\ Massachusetts Institute of Technology, Cambridge, Massachusetts
}

(Received 17 November 1998; accepted 14 January 1999)

\begin{abstract}
The question of how to proceed toward ever more realistic plasma simulation studies using ever increasing computing power is addressed. The answer presented here is the M3D (Multilevel 3D) project, which has developed a code package with a hierarchy of physics levels that resolve increasingly complete subsets of phase-spaces and are thus increasingly more realistic. The rationale for the multilevel physics models is given. Each physics level is described and examples of its application are given. The existing physics levels are fluid models (3D configuration space), namely magnetohydrodynamic (MHD) and two-fluids; and hybrid models, namely gyrokinetic-energetic-particle/MHD (5D energetic particle phase-space), gyrokinetic-particle-ion/ fluid-electron (5D ion phase-space), and full-kinetic-particle-ion/fluid-electron level (6D ion phase-space). Resolving electron phase-space (5D or $6 \mathrm{D})$ remains a future project. Phase-space-fluid models are not used in favor of $\delta f$ particle models. A practical and accurate nonlinear fluid closure for noncollisional plasmas seems not likely in the near future. (C) 1999 American Institute of Physics. [S1070-664X(99)93005-7]
\end{abstract}

\section{INTRODUCTION}

For the study of magnetized plasmas, as for other scientific research areas, three complementary tools are available: experiment, analytic theory, and numerical simulation. In recent years, as computers become ever more powerful, the importance of numerical simulation is widely being recognized and promoted. However, how to proceed toward ever more realistic simulation studies as computing power increases is an important question to be answered. In this paper, we present our answer to this question in the context of magnetic confinement fusion research.

Simulation of plasmas presents many difficulties. It is a numerically stiff problem, since plasma behavior contains wide ranges of length and time scales, e.g., the resistive time scales of present day large fusion experiments are on the order of seconds, while the ion and electron cyclotron periods are on the order of nanoseconds and picoseconds, respectively. It is also strongly anisotropic, e.g., heat conduction along magnetic field lines is more than $10^{10}$ times larger than that across field lines. Moreover, velocity space effects, such as wave-particle resonances, are often important due to infrequent collisions.

These and other difficulties preclude the possibility of simulations including all the relevant physics, at least for the near future. Approximate models are therefore used, and more and more realistic studies should be performed as computational capabilities and the understanding of plasmas in-

\footnotetext{
*Paper K6I1.6 Bull. Am. Phys. Soc. 43, 1810 (1998).

${ }^{\dagger}$ Invited speaker.

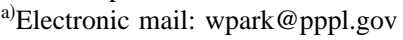

crease. Most current 3D (three dimensional) simulations are global simulations using the MHD (magnetohydrodynamic) model, which assumes collisional plasmas, or turbulence simulations using the electrostatic approximation where perturbed magnetic fields are neglected.

To determine the best strategy for more realistic simulations, we note that the key factor that determines the degree of realism and also the corresponding computational requirements is the phase-space resolved in the simulation. Thus, multilevel physics codes which resolve increasingly larger phase-spaces and are thus increasingly more realistic, can be built, and higher levels can be added as computing capabilities increase. Each existing physics level is also useful, because lower levels with less phase-space resolved are computationally more efficient, and more importantly, because higher level results with more complex physics must be compared to lower level results for the delineation of physics and to ascertain the basic validity of the higher level results.

Thus, in the M3D (Multilevel 3D) Project, ${ }^{1}$ we have built a code package which solves a hierarchy of physics levels with increasing realism. The existing physics levels which have been used in applications are fluid models (3D configuration space), namely $\mathrm{MHD}^{2}$ and two-fluids; ${ }^{3}$ and hybrid models, namely gyrokinetic-energetic-particle/MHD ${ }^{4,5}$ (5D energetic particle phase-space), gyrokinetic-particleion/fluid-electron ${ }^{6}$ (5D ion phase-space), and full-kineticparticle-ion/fluid-electron level ${ }^{7}$ (6D ion phase-space). At the present, electrons are described by fluid models only, because resolving electron phase-space (5D or 6D) at the level of the electron inertial length (skin depth) is not yet feasible 
for most plasma regimes of interest, and remains a future project.

Phase-space-fluid models such as a Vlasov fluid are not used in the M3D project in favor of $\delta f$ particle models. In high dimensional simulations, $\delta f$ particle models are much more efficient than phase-space-fluid models. To understand this, let us think about a specific case in which the structure lines up with one coordinate. For an error of $\epsilon$, the required number of particles scales as $1 / \epsilon^{2}$ because the random noise varies as the inverse square root of the particle number; the requisite fluid grid number scales as $1 / \epsilon^{n}$, where $n$ is the dimensionality of the model. This assumes a relatively uniform mesh for a phase-space-fluid model. With a nonuniform mesh or, more efficiently, with an unstructured mesh, the situation will be better. However, a particle model also has characters similar to the ultimately efficient unstructured mesh. For example, near a trapped-passing boundary, a single particle will describe the correct behavior as long as the fields in the 3D configuration space are well resolved. A phase-space-fluid model would require, in addition, a finely resolved grid in velocity space. When the perturbation is very small, a full- $f$ particle model becomes inefficient because the random noise is proportional to $f$. The $\delta f$ method $^{8}$ solves this problem by making the random noise proportional to the perturbation $(\delta f)$. (The $\delta f$ method, however, is inferior to a full- $f$ method when $\delta f \sim f$, so the optimal strategy switches back to a full- $f$ scheme when the perturbation grows.)

In addition to the multilevel physics structure, the M3D code has multilevel structures in the mesh scheme (structured and unstructured mesh options), ${ }^{9}$ geometry, boundary conditions, etc., so that it can be applied to a wide range of plasma states.

The paper is organized as follows. In the next section, the rationale is given for the actual multilevel physics models, and in Sec. III, each physics model is described and examples of its application are given. Section IV gives brief remarks on topics, such as unstructured mesh, parallelization, and future plans. The final section gives the summary.

\section{RATIONALE FOR MULTILEVEL PHYSICS MODELS}

From the kinetic equation,

$$
\frac{\partial f}{\partial t}+\mathbf{v} \cdot \frac{\partial f}{\partial \mathbf{x}}+\frac{\mathbf{F}}{m} \cdot \frac{\partial f}{\partial \mathbf{v}}=\left(\frac{\partial f}{\partial t}\right)_{c},
$$

velocity moments are taken to produce fluid equations. The main advantage of a fluid model is its lower dimensionality. However, the difficult question of closure remains for plasmas with low collision frequency.

To gain some insight, let us look at the exact total momentum equation,

$$
\rho \frac{d \mathbf{v}}{d t}=-\nabla \cdot \mathbf{P}+\mathbf{J} \times \mathbf{B} .
$$

If we assume that the exact pressure tensor $\mathbf{P}$ could be given, essentially all the relevant information is given by this momentum equation together with the electron momentum equation $\left(\mathbf{P}_{e}=0\right.$ and $\eta=0$ are assumed for this discussion), continuity equation, and electromagnetic field equations. (Moments higher than the divergence of the heat flux are not determined, but they usually do not have practical importance.) In this sense, the various closure schemes described below can be interpreted as ways to find an approximate $\mathbf{P}$.

The simplest fluid model, MHD, approximates the plasma as a single collisional conducting fluid, even though the actual plasma often is collisionless over the time scale of interest. The pressure becomes a scalar $p$ and the equation for $p$ is closed by the heat flux $\mathbf{q}=0$, following the assumption of high collision frequency. In spite of these approximations, the MHD model is often successful in explaining global phenomena, in large part due to the fact that small gyroradius size gives the plasma a collisional fluid-like behavior perpendicular to the field lines. However, it fails in many situations, such as when parallel dynamics or waveparticle resonances are important.

More sophisticated fluid models attempt more accurate closures. Let us write

$$
\mathbf{P}=p \mathbf{I}+\Pi_{i i}\left(p_{\|}-p_{\perp}\right)+\Pi_{g} .
$$

An approximate closure involving the last term, gyroviscosity, gives ion drift terms in the two-fluid momentum equation. ${ }^{10,11}$ The "neoclassical closure", is usually used to approximate $\hat{\mathbf{b}} \cdot \nabla \cdot \Pi_{i i}$, which is responsible for bootstrap current and neoclassical tearing modes. ${ }^{12,13}$

Still more accurate would be to time evolve $p_{\|}$and $p_{\perp}$ separately, as in the double adiabatic theory. ${ }^{14}$ To recover collision-time-scale phenomena such as the bootstrap current, collision terms should appear explicitly. A closure scheme that includes linear wave-particle resonances uses the heat flux in the form of $\mathbf{q}\left(k_{\|}\right)$to close the pressure equations. ${ }^{15}$ Since the wave-particle resonance is a nonlocal interaction, such a nonlocal closure involving a wave vector can be expected. Such a nonlocal closure is difficult, but possible to implement in an electromagnetic simulation via spectral analysis along field lines. However, a nonlinear closure which correctly gives nonlinear wave-particle interactions would be exceedingly difficult, if not impossible to implement, e.g., a formal closure scheme can use the heat flux in the form of $\mathbf{q}\left(k_{\|}, \omega\right) .{ }^{16}$ As can be expected, it is also temporally nonlocal, making implementation impractical. There are other efforts ${ }^{17}$ for better nonlinear closures and any improvement on nonlinear closure would be an important progress. However, we must conclude at this point that the possibility of a practical and accurate nonlinear closure in the near future is not good. This necessitates the next physics level, particle/fluid hybrid models. (Phase-space-fluid models are not considered for the reasons given in the Introduction.)

The simplest particle/fluid hybrid model is the gyrokinetic-energetic-particle/MHD hybrid scheme. ${ }^{4}$ When small numbers of energetic particles, such as fusion alphas, are present, their density can be neglected, while their thermal content can be included through the pressure tensor $\mathbf{P}_{h}$. In this scheme, the energetic particles are represented by particles following the gyrokinetic equations, ${ }^{18}$ and their pressure $\mathbf{P}_{h}$ is coupled to Eq. (2) by $\mathbf{P}=p+\mathbf{P}_{h}$, where $p$ is the bulk plasma scalar pressure. This scheme can be called a particle closure, and correctly gives nonlinear wave-particle 
interactions for energetic particles. Since gyrokinetic equations are used, the largest phase-space resolved is the 5D energetic particle phase-space. An alternate scheme couples to Eq. (2), using the energetic particle current instead of the pressure, calculated from the particle component, and is called "current coupling" scheme in contrast to "pressure coupling" given above.

The next physics level is the gyrokinetic-particle-ion/ fluid-electron model, where the bulk ions are also represented by gyrokinetic particles. ${ }^{6}$ It couples to Eq. (2) through both the pressure $\mathbf{P}$ and the density $\rho$ calculated from the ion particles. The computational requirement to resolve the $5 \mathrm{D}$ ion phase-space is comparable to the case of the $5 \mathrm{D}$ energetic particle phase-space, because the bulk ion phase-space is smaller, but finer resolution is required. This level also has a current coupling scheme in addition to the pressure coupling scheme.

The next physics level is the full-kinetic-ion/fluidelectron model, where the full $6 \mathrm{D}$ ion phase-space is resolved. This scheme had been known for many years, and had been used for space plasmas and FRC (Field Reversed Configuration) experiments where $\omega_{c i} \sim \omega_{A}$, often with reduced configurational space dimensions. Even with present day computational capabilities, full $6 \mathrm{D}$ studies in a $\omega_{c i}$ $\gg \omega_{A}$ regime are difficult, and we are currently applying this level to FRC studies.

Considering the fact that most previous global nonlinear simulations are done using the MHD model, it would be useful to have the concept of extended-MHD or XMHD, which can denote collectively the physics models which will most likely be used in the present to near future time scale. In a narrow sense, as used above, MHD means a single magneto-fluid model with collisional closure. In a broader sense, MHD (magnetohydrodynamics) is sometimes used to denote a general megneto-fluid model, such as our two-fluid model with an approximate nonlocal closure. XMHD then naturally denote a model which uses a megneto-fluid model at least as a component of the whole model, such as our hybrid models described above. Of course, an XMHD model should be realistic enough to include the three MHD waves. The models listed in the next section, which are all XMHD models, neglect some electron physics such as the nonlinear electron wave-particle interactions. Including these by resolving electron phase-space (either 5D or 6D) at the level of the electron inertial length is not yet feasible for most plasma regimes of interest, and remains a future project.

\section{MULTILEVEL PHYSICS MODELS}

This section lists each of the presently existing multilevel physics models of the M3D project, and describes some examples of its application.

\section{A. MHD model}

The dissipative MHD equations (in rationalized emu units) are used,

$$
\frac{\partial \mathbf{B}}{\partial t}=\nabla \times(\mathbf{v} \times \mathbf{B}-\eta \nabla \times \mathbf{B}),
$$
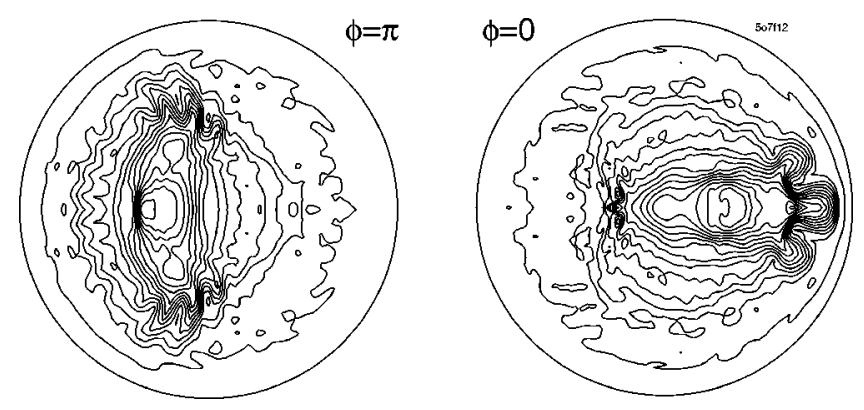

FIG. 1. Pressure contours during a high- $\beta$ disruption for a reversed shear plasma. Nonlinear development of a ballooning mode produces pressure steepening in the normal shear region with a structure which looks like fingers in $2 \mathrm{D}$ and ribbons in $3 \mathrm{D}$. This can cause magnetic field line stochasticity and a thermal quench.

$$
\begin{aligned}
& \rho \frac{d \mathbf{v}}{d t}=-\nabla p+(\nabla \times \mathbf{B}) \times \mathbf{B}+\mu \nabla^{2} \mathbf{v}, \\
& \frac{\partial \rho}{\partial t}=-\nabla \cdot \rho \mathbf{v}, \\
& \frac{\partial T}{\partial t}=-\mathbf{v} \cdot \nabla T-(\gamma-1) T \nabla \cdot \mathbf{v}+\kappa_{\perp} \nabla^{2} T .
\end{aligned}
$$

Additional wave equations for the temperature are used to represent the fast thermal equilibration along field lines, ${ }^{19}$

$$
\begin{aligned}
& \frac{\partial T}{\partial t}=\frac{s}{\rho} \mathbf{B} \cdot \nabla u, \\
& \frac{\partial u}{\partial t}=s \mathbf{B} \cdot \nabla T-\nu \nabla^{2} u .
\end{aligned}
$$

The wave speed, a constant $s$ times $B / \rho^{1 / 2}$ (the Alfven speed), represents the free streaming velocity of electrons (when electron temperature is more important) with a dissipation $\nu$. The thermal energy $\int \rho T d \tau$ inside a flux tube is conserved, and the "wave energy," $\frac{1}{2} \int\left(\rho T^{2}+u^{2}\right) d \tau$ is also conserved except for the dissipation due to $\nu$. The asymptotic state satisfies $\mathbf{B} \cdot \nabla T=0$. In this MHD level, the ambipolar electric field effect on electron temperature is neglected, and Eqs. (8) and (9) are valid on a time scale long compared to the electron-electron collision time. This wave representation of thermal equilibration along the field line is, for most experiments with small collision frequency, physically at least as accurate as the usual dissipative representation and is more accurate in numerical implementation.

The MHD applications include studies of the high- $\beta$ disruption; in normal shear ${ }^{2}$ and in reversed shear cases ${ }^{1}$ (shown in Fig. 1), and double tearing sawteeth. ${ }^{20}$

\section{B. Two-fluid model}

The two-fluid equations are obtained by generalizing the perturbative drift ordering to arbitrary perturbation size. ${ }^{3}$ They are closely related, although not identical, to the collisional Braginskii equations. ${ }^{21}$ The model was chosen, in part, to transform smoothly into the resistive MHD equations in the limit of vanishing gyroradii. 
The drift ordering ${ }^{11}$ assumes fluid velocities and growth rates small compared to the thermal velocity scales of the MHD ordering, $v / v_{t h} \sim \delta, \partial / \partial t \sim \delta v_{t h} / L$, and $\omega / \omega_{c i} \sim \rho_{i} / L$ $\sim \delta$, where $\delta$ is a characteristic small parameter, $v_{t h}$ is the ion thermal speed, $L$ a characteristic equilibrium scale length, $\omega$ a typical frequency, $\omega_{c i}$ the ion cyclotron frequency, and $\rho_{i}$ the ion gyroradius. The ordering introduces the diamagnetic velocities

$$
\begin{aligned}
& \mathbf{v}_{* j}=\mathbf{B} \times \nabla p_{j} /\left(q_{j} n_{j} B^{2}\right), \\
& \mathbf{v}_{* T j}=\mathbf{B} \times \nabla T_{j} /\left(q_{j} B^{2}\right),
\end{aligned}
$$

for $j=e, i$, where $q_{j}$ is the particle charge.

The fluid velocities can be written exactly as

$$
\begin{aligned}
& \mathbf{v}_{i}=\mathbf{v}+\mathbf{v}_{d i}, \\
& \mathbf{v}_{e}=\mathbf{v}+\mathbf{v}_{* e}-\mathbf{J}_{\|} / e n_{e}, \\
& \mathbf{v}=\mathbf{v}_{\perp}+\mathbf{v}_{i \|},
\end{aligned}
$$

where $\mathbf{v}_{\perp}$ is the perpendicular guiding center velocity of the electrons and ions, neglecting magnetic drifts. The generalized "diamagnetic" part $\mathbf{v}_{d i}$ of the ion fluid velocity perpendicular to the magnetic field is defined to be

$$
\mathbf{v}_{d i} \equiv \frac{\mathbf{J}_{\perp}}{e n_{e}}+\mathbf{v}_{* e},
$$

where $\mathbf{v}_{* e}$ is given by Eq. (10) with $j=e$.

In rationalized emu units, the essential features of our two-fluid model can be summarized as

$$
\begin{aligned}
& \frac{\partial \mathbf{v}}{\partial t}+(\mathbf{v} \cdot \nabla) \mathbf{v}=-\left(\mathbf{v}_{d i} \cdot \nabla\right) \mathbf{v}_{\perp}+\frac{\mathbf{J} \times \mathbf{B}}{n m_{i}}-\frac{\nabla p}{n m_{i}} \\
& -\frac{\hat{\mathbf{b}}}{n m_{i} B} \mathbf{B} \cdot \nabla \cdot \Pi_{i}+\mu \nabla^{2} \mathbf{v}_{i}, \\
& \mathbf{E}+\mathbf{v} \times \mathbf{B}=\eta \mathbf{J}-\frac{\nabla_{\|} p_{e}}{e n}-\frac{\hat{\mathbf{b}}}{n e B} \mathbf{B} \cdot \nabla \cdot \Pi_{e}, \\
& \frac{\partial \mathbf{B}}{\partial t}=-\nabla \times \mathbf{E}, \quad \mathbf{J}=\nabla \times \mathbf{B}, \quad 0=\nabla \cdot \mathbf{B}, \\
& \frac{\partial p_{i}}{\partial t}+\mathbf{v}_{i} \cdot \nabla p_{i}=-\gamma_{i} p_{i} \nabla \cdot \mathbf{v}_{i}+\nabla \cdot n \kappa_{\perp i} \nabla_{\perp}\left(\frac{p_{i}}{n}\right) \\
& +\nabla \cdot n \kappa_{\| i} \nabla_{\|}\left(\frac{p_{i}}{n}\right)-\gamma_{i} \nabla \cdot\left(p_{i} \mathbf{v}_{* T i}\right), \\
& \frac{\partial p_{e}}{\partial t}+\mathbf{v}_{e} \cdot \nabla p_{e}=-\gamma_{e} p_{e} \nabla \cdot \mathbf{v}_{e}+\nabla \cdot n \kappa_{\perp e} \nabla_{\perp}\left(\frac{p_{e}}{n}\right) \\
& +\nabla \cdot n \kappa_{\| e} \nabla_{\|}\left(\frac{p_{e}}{n}\right)-\gamma_{e} \nabla \cdot\left(p_{e} \mathbf{v}_{* T e}\right),
\end{aligned}
$$

$$
\frac{\partial n}{\partial t}+\mathbf{v}_{e} \cdot \nabla n=-n \nabla \cdot \mathbf{v}_{e} .
$$

The neoclassical parallel viscosity terms involving $\Pi_{i}$ and $\Pi_{e}$ are approximated using the usual neoclassical closure. ${ }^{12,13}$ The electron mass is neglected and quasineutral-

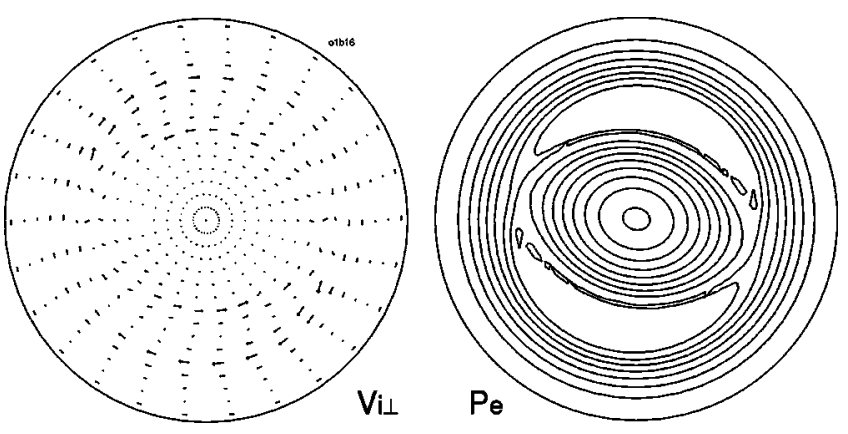

FIG. 2. Profiles with steady state, bootstrap current driven neoclassical magnetic islands. The left figure shows that the perpendicular flow of ion fluid vanishes except near the island region, as expected in this $\nabla T=0$ case. The islands (shown here as flat electron pressure regions) rotate with $\omega$ $\simeq 0.3 \omega_{e}^{*}$ in the lab frame, and $\omega \simeq 0.7 \omega_{i}^{*}$ in the guiding center frame. Without the neoclassical effects, the islands are fixed in the guiding center frame.

ity, $n_{e}=n_{i}=n$, is assumed. Here $p_{j}=n T_{j}, p$ is the total pressure. The $\gamma_{j}$ 's are the ratios of the specific heats. Although written above in a dissipative form, for convenience, the effect of large $\kappa_{\| j}$ is actually modeled using a wave representation similar to the one described for MHD.

Figure 2 shows an example which studies the rotation of bootstrap current driven neoclassical magnetic islands.

\section{Gyrokinetic-energetic-particle/MHD hybrid model}

To model the nonlinear interaction of energetic particles with MHD waves, a hybrid particle/MHD model has been developed. ${ }^{4}$ A small energetic to bulk ion density ratio was assumed, such that the energetic ion perpendicular inertia in the momentum equation can be neglected. The plasma is divided into two parts: the bulk plasma, which contains the thermal electrons and ions, and the energetic hot ions. The bulk plasma is described by the ideal MHD equations, whereas the hot ions are described by the gyrokinetic equations. ${ }^{18}$ The particle part can be coupled to the bulk plasma part through one of two almost equivalent, accurate coupling schemes, pressure coupling or current coupling. In the pressure coupling scheme, the hot particle pressure tensor $\mathbf{P}_{h}$ is coupled to the bulk plasma momentum equation,

$$
\rho_{b} \frac{d \mathbf{v}_{b}}{d t}=-\nabla p_{b}-\left(\nabla \cdot \mathbf{P}_{h}\right)_{\perp}+\mathbf{J} \times \mathbf{B} .
$$

In the current coupling scheme, the hot particle current density $\mathbf{J}_{h}$ and charge density $q_{h}$ are coupled to the bulk plasma momentum equation

$$
\rho_{b} \frac{d \mathbf{v}_{b}}{d t}=-\nabla p_{b}+\left(\nabla \times \mathbf{B}-\mathbf{J}_{h}\right) \times \mathbf{B}+q_{h} \mathbf{v}_{b} \times \mathbf{B},
$$

where the subscript $b$ denotes the bulk part and the subscript $h$ denotes the hot ion component. The last term of Eq. (23) can be thought of as the subtraction of the $\mathbf{J} \times \mathbf{B}$ force on the electrons whose density is the same as the hot particles. This term cannot be neglected because the $\mathbf{E} \times \mathbf{B}$ drift can be comparable to the perturbation of the magnetic drift of the hot particles. 

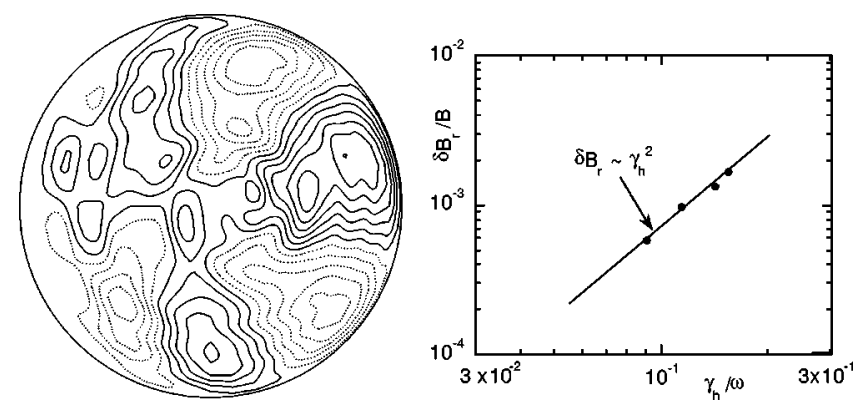

FIG. 3. The left figure shows the stream lines of the incompressible part of the velocity in a nonlinearly saturated TAE mode. The saturation mechanism is found to be wave-particle trapping. The right figure shows the saturation amplitude as a function of the growth rate, and agreement with the analytic prediction.

Equations (22) or (23), together with the other MHD equations, form the MHD part which is advanced in time using particle quantities given by the particle part. The new $\mathbf{E}$ and $\mathbf{B}$ are in turn used by the particle part to advance the particle quantities in time. The model is fully self-consistent, including nonlinear wave-particle resonances and other hot particle interactions with MHD waves, and the nonlinear MHD mode coupling.

The ions are pushed using the guiding center equations of motion

$$
\begin{aligned}
& \dot{\mathbf{X}}=\frac{1}{B}\left[\mathbf{B}^{*} U+\hat{\mathbf{b}} \times(\mu \nabla B-\mathbf{E})\right], \\
& \dot{U}=-\frac{1}{B} \mathbf{B}^{*} \cdot\left(\mu \nabla B-\frac{e}{m} \mathbf{E}\right), \quad \dot{\mu}=0,
\end{aligned}
$$

where $(\mathbf{X}, U, \mu, \theta)$ are gyrocenter coordinates, and $\mathbf{B}^{*}=\mathbf{B}$ $+(m / e) U \hat{\mathbf{b}} \times(\hat{\mathbf{b}} \cdot \nabla \hat{\mathbf{b}})$. The equilibrium distribution function, $F_{0}=F_{0}\left(p_{\phi}, \mu, \epsilon\right)$, is a function of the integrals of motion: $p_{\phi}=R \hat{\phi} \cdot \mathbf{A}^{*}=e \psi+m U R b_{\phi}$ (toroidal angular momentum) and $\epsilon=\mu B+m U^{2} / 2$.

When perturbation amplitudes are small, such as in a linear run, the $\delta f$ method is used to reduce particle noise.

This hybrid scheme has been used by several groups to study torodal Alfreń eigenmodes (TAE) modes, fishbone oscillations, and space plasmas. Some earlier examples are: Ref. 5 using the pressure coupling scheme, Refs. 22 and 23 using the current coupling scheme, and Ref. 24 using the pressure coupling scheme with the reduced MHD equations. Figure 3 shows the nonlinear saturation of nonoverlapping TAE modes. ${ }^{5}$ The saturation mechanism was found to be particle trapping in nonlinear waves. Thus, for this study, a fluid model with linear Landau closure would not have been sufficient.

\section{Gyrokinetic-particle-ion/fluid-electron hybrid model}

To include ion particle-wave resonances, ion gyroviscosity, and neoclassical effects more self-consistently, the bulk ion phase-space can be resolved using gyrokinetic particle ions. As in the previous level, both pressure and current coupling schemes are presented.

\section{Pressure coupling scheme}

In the pressure coupling scheme, ${ }^{6}$ the ion fluid velocity is calculated by solving the momentum equation, and the calculated ion fluid velocity is used in the Ohm's law. Quasineutrality is assumed,

$$
\begin{aligned}
& \frac{\partial\left(\rho_{i} \mathbf{V}_{i}\right)}{\partial t}=-\nabla p_{e}-\boldsymbol{\nabla} \cdot \mathbf{P}_{i}^{\mathrm{CGL}}-\boldsymbol{\nabla} \cdot \boldsymbol{\Pi}_{g i}+\mathbf{J} \times \mathbf{B} \\
& \partial \mathbf{B} / \partial t=-\boldsymbol{\nabla} \times \mathbf{E}, \\
& \mathbf{E}=-\mathbf{V}_{e} \times \mathbf{B}-\frac{1}{e n_{e}} \boldsymbol{\nabla} p_{e}-\frac{\hat{\mathbf{b}}}{n e B} \mathbf{B} \cdot \nabla \cdot \Pi_{e} .
\end{aligned}
$$

Here the ion pressure is taken to be in CGL (Chew, Goldberger, Lowe) form and the gyroviscosity part of the stress tensor is calculated in order to take into account the diamagnetic effects. The ion pressure is calculated from the gyrokinetic particles, whereas the gyroviscosity tensor, $\boldsymbol{\Pi}_{g}$, is either calculated from the particles as shown below, or from a fluid closure similar to Refs. 10 and 11 .

The gyroviscosity terms appear naturally in the gyrokinetic description, when the transformation from the gyrocenter to particle coordinates is made in the pressure integral; however, this approach requires that the ion gyroradius be resolved in the simulations. In order to include the diamagnetic effects in the drift-kinetic formulation, a small ion gyroradius expansion can be made in the pressure tensor integral, which gives the expression for the gyroviscosity stress tensor in terms of the gyrofluid moments (with $m=e=1$ ),

$$
\begin{aligned}
\mathbf{P}_{i}^{\mathrm{CGL}}= & \left\|U^{2}\right\| \hat{\mathbf{b}} \hat{\mathbf{b}}+\|\mu B\|(\mathbf{I}-\hat{\mathbf{b}} \hat{\mathbf{b}}), \\
\boldsymbol{\nabla} \cdot \boldsymbol{\Pi}_{g i}= & \hat{\mathbf{b}} \times \boldsymbol{\nabla}\left(\nabla_{\|}\|\mu U\|\right)+\nabla_{\perp}\left(\nabla_{\perp}^{2}\left\|\mu^{2}\right\| / 4-3 \chi_{\perp} / 2\right. \\
& \left.+n \mathbf{V}_{* i} \cdot \mathbf{V}_{E}\right)+\nabla_{\|}\left(\nabla_{\perp}^{2}\left\|U^{2} \mu\right\| / 2 B-\chi_{\|}-\chi_{\perp}\right) \\
& +\nabla \cdot\left(n \mathbf{V}_{E} \mathbf{V}_{E}\right),
\end{aligned}
$$

where

$$
\begin{aligned}
& \chi_{\perp(\|)}=-\frac{p_{\perp(\|)}}{B} \hat{\mathbf{b}} \cdot \nabla \times \mathbf{V}_{E}, \quad\|*\|=\int(*) F_{i} d^{3} \mathbf{v}, \\
& F_{i}=F(\mathbf{X}, U, \mu, t), \quad \hat{\mathbf{b}}=\mathbf{B} / B .
\end{aligned}
$$

The above expressions were obtained by performing the transformation from particle variables to guiding-center coordinates in the stress tensor integral and then expressing the guiding-center distribution function in terms of the gyrocenter distribution function $F$. After gyroaveraging, a small $\left(k_{\perp} \rho\right)$ expansion was made, using the ordering

$$
\frac{\rho_{i}}{L} \sim \frac{\omega}{\omega_{c i}} \sim \frac{k_{\|}}{k_{\perp}} \sim \varepsilon, \quad \varepsilon \leqslant\left(k_{\perp} \rho\right)^{2}<1 .
$$

Here $\mathbf{P}^{\mathrm{CGL}}$ is the zero order (in $\varepsilon$ ) part of the ion stress tensor $\mathbf{P}$ and gyroviscosity tensor $\boldsymbol{\Pi}_{g}$ is defined here to include FLR corrections to $\mathbf{P}$ [up to $O\left(k_{\perp}^{2} \rho^{2}\right)$ ] plus the inertial term: $\boldsymbol{\Pi}_{g} \equiv \mathbf{P}-\mathbf{P}^{\mathrm{CGL}}$. The diagonal corrections, which represent the difference between the gyrofluid moments and the particle-fluid moments are also taken into account in $\boldsymbol{\Pi}_{g}$. 
The gyroviscosity part of the stress tensor was derived assuming an electrostatic perturbation and a uniform background magnetic field.

\section{Current coupling scheme}

(This scheme is not yet implemented in the code.) The current coupling scheme ${ }^{25}$ at this level is structurally similar to the full-kinetic-particle-ion/fluid-electron hybrid level given in the next section. The main difference, in addition to the fact that the particles are followed using the gyrokinetic equation instead of the basic equation of motion, is that the ion polarization current has to be added explicitly to the ion current of gyrokinetic particles to find the total ion current, Eq. (34). (This explicit addition would become unnecessary, if a gyrokinetic formalism can be found through which the ion polarization current can be obtained with enough numerical accuracy.) From the total ion current and total current $\mathbf{J}$ $=\nabla \times \mathbf{B}$, electron current and velocity are found, Eq. (31). The electron velocity, together with the electron momentum equation and the electromagnetic field equations, determine the electromagnetic fields at the next time step. These fields in turn are used to advance the gyrokinetic particle ions.

$$
\begin{aligned}
& \mathbf{v}_{e}=\frac{1}{n e}\left(\mathbf{J}_{i}-\mathbf{J}\right), \quad \mathbf{J}=\nabla \times \mathbf{B}, \\
& \mathbf{E}=-\mathbf{v}_{e} \times \mathbf{B}-\frac{1}{n e} \nabla p_{e}-\frac{\hat{\mathbf{b}}}{n e B} \mathbf{B} \cdot \nabla \cdot \Pi_{e}, \\
& \frac{\partial \mathbf{B}}{\partial t}=-\nabla \times \mathbf{E}, \\
& \mathbf{J}_{i}=\mathbf{J}_{G K}+\mathbf{J}_{p}, \\
& \mathbf{J}_{p}=\frac{n}{\omega_{c i} B} \frac{d \mathbf{E}}{d t}, \\
& \frac{d}{d t}=\frac{\partial}{\partial t}+\mathbf{v}_{i} \cdot \nabla .
\end{aligned}
$$

Both the current coupling and the pressure coupling schemes give, as expected, the following dispersion equation in the limit of small $k_{\perp} \rho_{i}$ and low $\beta$ in a slab:

$$
\begin{aligned}
& {\left[\omega\left(\omega-\omega_{i}^{*}\right)-k_{\|}^{2} v_{A}^{2}\right]\left[\omega\left(\omega-\omega_{i}^{*}\right)-k^{2} v_{A}^{2}\right.} \\
& \left.\quad-\frac{2 k_{\perp}^{2} T_{i}}{n_{o} m_{i}} \int \frac{\left(\omega-\omega_{i}^{*}\right) F_{o i}}{\omega-k_{\|} u} d u d \mu\right]=\left(\omega_{c i} \omega_{i}^{*}\right)^{2} .
\end{aligned}
$$

\section{Application}

Figure 4 shows a study in which three different levels of M3D multilevel physics models were used. Growth rates (a) and rotation frequencies (b) of $m=1$ internal modes at various $\beta_{h}$ values of fusion alphas are shown. Triangles are obtained from MHD, and show that when $\beta_{h}=0$, the system is unstable to the $m=1$ mode. Solid circles are obtained from the gyrokinetic-energetic-particle/MHD hybrid model, and show that as $\beta_{h}$ is increased, the $m=1$ mode is first stabilized, but at higher $\beta_{h}$, a new alpha particle driven mode, fishbone oscillations, is destabilized with rotation frequency
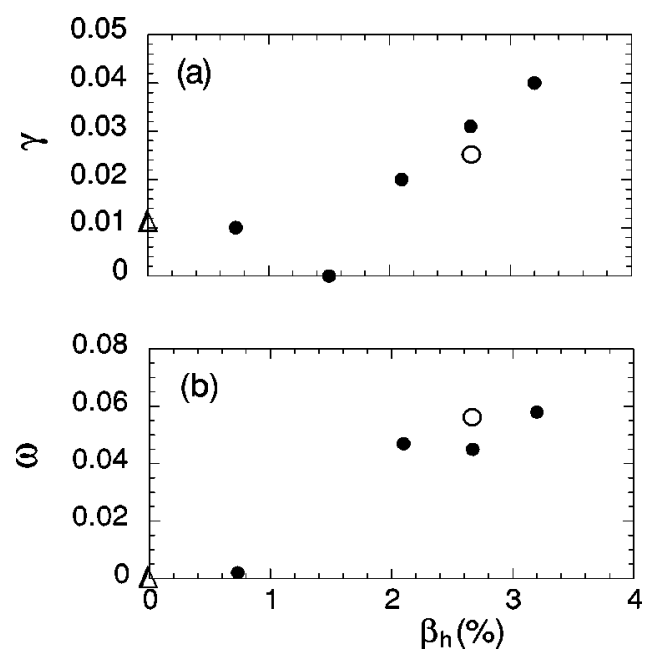

FIG. 4. (a) Growth rates, and (b) rotation frequencies of $m=1$ internal modes at various $\beta_{h}$ values of the fusion alphas, obtained from MHD (triangles), from the gyrokinetic-energetic-particle/MHD hybrid model (solid circles), and from the gyrokinetic-particle-ion/fluid-electron hybrid model (open circles).

$\omega$ near the trapped alpha particle precession frequency, $\omega_{d, h \text {,trap }}$. Open circles are obtained from the gyrokineticparticle-ion/fluid-electron hybrid model and show that when fluid thermal ions are replaced by particles, the mode is slightly stabilized. This is because the passing thermal ions can resonate with the mode, $\omega_{t r, t h \text {,pass }} \sim \omega$ and the effect is stabilizing, since $\omega_{t h}^{*}<\omega$. The rotation frequency of the mode increases slightly with thermal ion particles. Figure 5 shows the profiles of the case corresponding to the open circles in Fig. 4.

\section{E. Full-kinetic-particle-ion/fluid-electron hybrid model}

The basic scheme is similar to, but simpler than, the current coupling scheme of the previous level. Now the total ion current can be calculated directly from particles following the basic equation of motion.

$\frac{d \mathbf{v}_{i}}{d t}=\frac{q_{i}}{m_{i}}\left(\mathbf{E}+\mathbf{v}_{i} \times \mathbf{B}\right)+[$ collisions $]$,

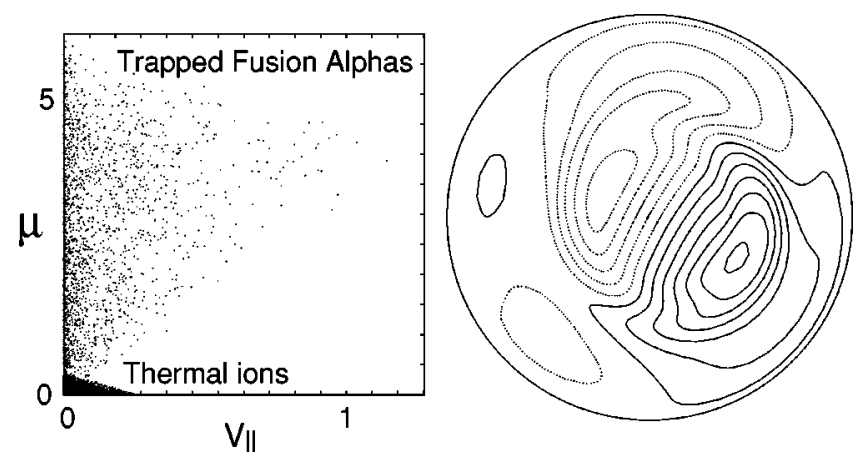

FIG. 5. The profiles of the case corresponding to the open circles in Fig. 4. The magnetic moment $\mu$ versus $v_{\|}$plot on the left shows the trapped part of the slowing down fusion alphas, and thermal ion particles with a temperature 0.01 times the alpha temperature. A quarter million particles each are used to represent the alphas and thermal ions. The figure on the right shows the stream lines of the incompressible part of the mass flow. 

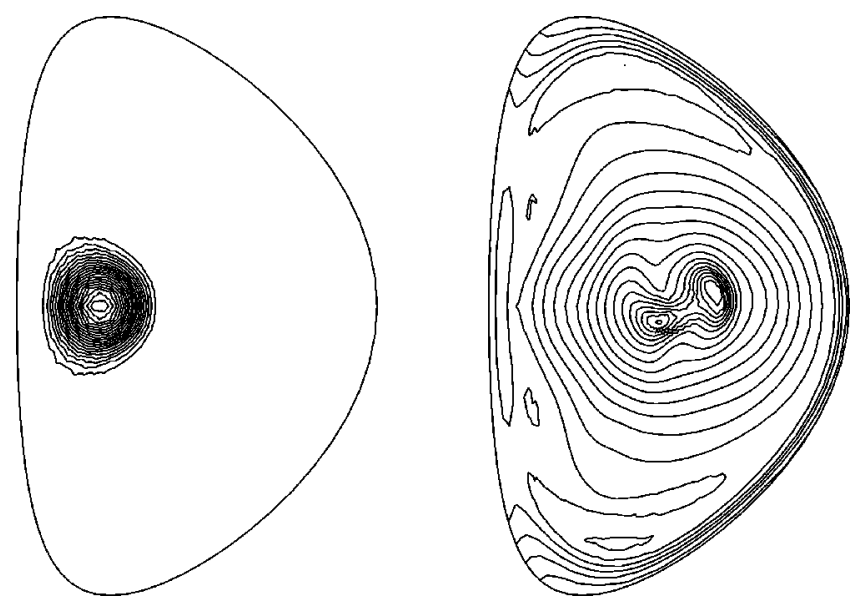

FIG. 6. Density contours showing an inboard-side injected pellet. The pellet can penetrate deep into the plasma, accompanying a reconnection process as shown on the right.

$$
\begin{aligned}
& \mathbf{v}_{e}=\frac{1}{n e}\left(\mathbf{J}_{i}-\mathbf{J}\right), \quad \mathbf{J}=\nabla \times \mathbf{B}, \\
& \mathbf{E}=-\mathbf{v}_{e} \times \mathbf{B}-\frac{1}{n e} \nabla p_{e}-\frac{\hat{\mathbf{b}}}{n e B} \mathbf{B} \cdot \nabla \cdot \Pi_{e}, \\
& \frac{\partial \mathbf{B}}{\partial t}=-\nabla \times \mathbf{E} .
\end{aligned}
$$

This scheme has been known for many years, and has been used for space plasmas and FRC experiments where $\omega_{c i}$ $\sim \omega_{A}$, often with reduced configurational space dimensions. Even with present day computational capabilities, the full 6D studies in the $\omega_{c i} \gg \omega_{A}$ regime are difficult, and we are currently applying this level to FRC studies.

\section{SPATIAL REPRESENTATION AND OTHER TOPICS}

Due to a limitation on the length of the paper, this paper concentrates on physics models, and only briefly covers other critical topics, such as spatial representation, computational issues like parallelization, and future work plans.

In addition to realistic physics models, a realistic simulation requires resolution of multiscale spatial structures, such as reconnection layers, and a realistic representation of geometric effects and machine hardware such as the resistive wall. For an efficient representation of these effects, a finite element unstructured mesh option is available for MHD and two-fluid levels, and is expected to be extended to other levels. ${ }^{9}$ This version has been applied to study pellet injection, disruptions interacting with a resistive wall, and runaway electron generation. ${ }^{26}$ Figure 6 shows an inboardinjected-pellet simulation. Inboard injection is found to be more favorable than outboard injection, in agreement with recent Axisymmetric Divertor Experiment (ASDEX) results. ${ }^{27}$ The pellet can penetrate deep into the plasma, accompanying a reconnection process. This effect could be used for deep fueling of large tokamaks like International Thermonuclear Experimental Reactor (ITER), where a pellet is expected to ablate near the surface.
To deal with the complex structure of our project, two approaches are used: (1) to combine as many options as possible into one code using object oriented modular design, (2) when such a combination takes too much overhead, to maintain a separate code, keeping full interchangeability of the restart files. For the object oriented design, $\mathrm{C}++$ is used for the shell structure, while Fortran 77 and F90 are used for the core part for efficiency.

A coarse grain parallelization has been done on a sharedmemory 64 processor Origin2000, using OpenMP, and good scalability was obtained. ${ }^{28}$ For massively parallel, distributed memory computers, a scalable and efficient iterative matrix solver is being implemented.

Our near term plan on the physics side is to make existing physics levels more complete by adding the physics which is included in the models described before but not yet implemented. Most of the relevant ion physics is already included, except for collisions, which will be implemented in the near term. For the electron physics, inertia, double adiabatic type equations for parallel and perpendicular electron pressures with collision terms, and linear Landau damping will be implemented in the near term. In the longer term, the electron phase-space, first in 5D, should be resolved using gyrokinetic particles. (When gyrokinetic particles are implemented, drift-kinetic particles are also implemented utilizing the subset. Even with drift-kinetic particles, resolving tokamak plasmas at the level of the electron inertial length is not feasible for the near future, so 5D resolution of electron phase-space would be applied to other studies.) The whole project is difficult and long term, but fortunately, each step on the way is useful by itself. Obtaining physics results from the existing code is the most important aspect and should intensify as our codes mature and computational resources increase. Another important aspect is to make the code more user friendly.

\section{SUMMARY}

The question of how to proceed toward ever more realistic plasma simulation studies using ever increasing computing power is addressed. One answer is the M3D (Multilevel 3D) project, which has developed a code package with a hierarchy of physics levels that resolve increasingly complete subsets of phase-spaces and are thus increasingly more realistic. Each existing physics level is also useful, because lower physics levels with less phase-space resolved are computationally more efficient; and, more importantly, because higher level results with more complex physics must be compared to lower level results for the delineation of physics and to ascertain the basic validity of the higher level results.

The rationale for the multilevel physics models is given. Each physics model is described and examples of its application are given. The existing physics models are fluid models (3D configuration space), namely MHD and two-fluids; and hybrid models, namely gyrokinetic-energetic-particle/ MHD (5D energetic particle phase-space), gyrokineticparticle-ion/fluid-electron (5D ion phase-space), and fullkinetic-particle-ion/fluid-electron level (6D ion phase-space). An unstructured mesh option is also available for efficient 
representations of geometric effects. The examples of simulation studies given here are high- $\beta$ disruptions, bootstrap current driven neoclassical magnetic island rotation, nonlinear TAE mode saturation, fishbones, and pellet injection studies.

At present, electrons are described by fluid models only, because resolving electron phase-space (either $5 \mathrm{D}$ or $6 \mathrm{D}$ ) at the level of the electron inertial length is not yet feasible for most plasma regimes of interest, and remains a future project. A practical and accurate nonlinear fluid closure for noncollisional plasmas is not likely in the near future. In high dimensional simulations, $\delta f$ particle models are much more efficient than phase-space-fluid models, and therefore chosen for the hybrid models.

\section{ACKNOWLEDGMENTS}

This work was supported by the United States Department of Energy under Contract No. DE-AC02-76-CHO3073, Contract No. DE-FG02-91ER54109, and Grant No. DE-FG02-86ER53223.

${ }^{1}$ W. Park, Z. Chang, E. Fredrickson, G. Y. Fu, N. Pomphrey, H. R. Strauss, and L. E. Sugiyama, Plasma Physics and Controlled Nuclear Fusion Research (International Atomic Energy Agency, Vienna, 1997), Vol. 2, p. 411.

${ }^{2}$ W. Park, E. Fredrickson, A. Janos, J. Manickam, and W. M. Tang, Phys. Rev. Lett. 75, 1763 (1995).

${ }^{3}$ L. E. Sugiyama and W. Park, "Two-fluid Simulation Studies of Tokamak Plasmas," M.I.T. Research Laboratory of Electronics Report PTP-97/1, Phys. Plasmas (submitted).

${ }^{4}$ W. Park, S. E. Parker, M. Chance et al., Phys. Fluids B 4, 2033 (1992).

${ }^{5}$ G. Y. Fu and W. Park, Phys. Rev. Lett. 74, 1594 (1995).

${ }^{6}$ E. V. Belova, W. Park, G. Y. Fu, H. R. Strauss, and L. E. Sugiyama, in Proceedings of the Workshop on Nonlinear MHD and Extended MHD, Univ. of Wisconsin Center for Plasma Theory Report UW-CPTC-98-1
(1998), available from the National Technical Information Service, U.S. Department of Commerce, 5285 Port Royal Road, Springfield, Virginia 22161.

${ }^{7}$ E. V. Belova and W. Park, Bull. Am. Phys. Soc. 43, 1750 (1998).

${ }^{8}$ S. E. Parker and W. W. Lee, Phys. Fluids B 5, 77 (1993).

${ }^{9}$ H. R. Strauss and W. Park, Phys. Plasmas 5, 2676 (1998).

${ }^{10}$ Z. Chang and J. D. Callen, Phys. Fluids B 4, 1766 (1992)

${ }^{11}$ R. D. Hazeltine and J. D. Meiss, Phys. Rep. 121, 1 (1985); Plasma Confinement (Addison-Wesley, Redwood City, CA, 1992).

${ }^{12}$ S. P. Hirschman and D. J. Sigmar, Nucl. Fusion 9, 1079 (1981).

${ }^{13}$ J. D. Callen, W. X. Qu, K. D. Siebert et al., Plasma Physics and Controlled Nuclear Fusion Research (International Atomic Energy Agency, Vienna, 1986), Vol. 2, p. 157.

${ }^{14}$ G. F. Chew, M. L. Goldberger, and F. E. Low, Proc. R. Soc. London, Ser. A 236, 112 (1956).

${ }^{15}$ P. B. Snyder, G. W. Hammet, and W. Dorland, Phys. Plasmas 4, 3974 (1997).

${ }^{16}$ Z. Chang and J. D. Callen, Phys. Fluids B 4, 1167 (1992).

${ }^{17}$ N. Mattor, Phys. Plasmas 5, 1822 (1998).

${ }^{18}$ A. Brizard, J. Plasma Phys. 41, 541 (1989).

${ }^{19}$ W. Park, D. A. Monticello, H. Strauss, and J. Manickam, Phys. Fluids 29, 1171 (1986).

${ }^{20}$ Z. Chang, W. Park, E. D. Fredrickson et al., Phys. Rev. Lett. 77, 3553 (1996).

${ }^{21}$ S. I. Braginskii, in Reviews of Plasma Physics, edited by M. A. Leontovich (Consultants Bureau, New York, 1965), Vol. 1, p. 205.

${ }^{22}$ Y. Todo, T. Sato, K. Watanabe et al., Phys. Plasmas 2, 2711 (1995).

${ }^{23}$ E. V. Belova, R. E. Denton, and A. A. Chan, J. Comput. Phys. 136, 324 (1997).

${ }^{24}$ S. Briguglio, G. Vlad, F. Zonka, and C. Kar, Phys. Plasmas 2, 3711 (1995).

${ }^{25}$ E. V. Belova, W. Park, G. Y. Fu, H. R. Strauss, and L. E. Sugiyama, Bull. Am. Phys. Soc. 42, 1854 (1997).

${ }^{26}$ H. R. Strauss, W. Park, E. V. Belova, G. Y. Fu, and L. E. Sugiyama, in Proceedings of 17th Plasma Physics and Controlled Nuclear Fusion Research, Yokohama, 1997 (International Atomic Energy Agency, Vienna, 1998) Paper TH3/4.

${ }^{27}$ P. T. Lang, K. Buechl, M. Kaufmann et al., Phys. Rev. Lett. 79, 1487 (1997).

${ }^{28}$ X. Tang, W. Park, and H. R. Strauss, Bull. Am. Phys. Soc. 43, 1931 (1998). 